\title{
Computer simulation studies of Ar clusters ${ }^{\dagger}$
}

\author{
P PADMA KUMAR and K J RAO* \\ Solid State and Structural Chemistry Unit, Indian Institute of Science, Bangalore 560 012, India
}

\begin{abstract}
Results of the solid-liquid transition of $\mathbf{A r}_{13}$ cluster in a spherically symmetric external potential have been presented. The transition temperature is observed to show an elevation with pressure. The broadening of the heat capacity peaks indicate the transition becoming more diffused with pressure. The icosahedral structure of the cluster remains unaltered under pressure. $\mathbf{A r}_{55}$ cluster has also been studied by similar approach. A possible connection between glass transition phenomenon and melting of clusters under pressure has been examined.
\end{abstract}

Keywords. Computer simulation studies; Ar clusters.

\section{Introduction}

Discovery of unusual properties of nano-crystalline materials and small atomic clusters has spurred a great interest in the study of small aggregates of atoms and materials in the finely divided state ${ }^{\ddagger}$. Molecular dynamics and Monte Carlo simulation are methods by which properties of small clusters of atoms and molecules can be examined in detail, provided the interaction potentials are known at least fairly accurately (Etters and Kaelberer 1977; Jellinek et al 1986; Davis et al 1987; Labastie and Whetten 1990; Cheng et al 1992; Rose and Berry 1993; Frantz 1995). The unusual stability of isolated clusters of atoms, the magic numbers characterizing stable assemblies and geometries of the resulting aggregation which are often very highly symmetrical but non space filling (Chitra and Yashonath 1996; Häkkinen and Manninen 1996; Ikesyoji et al 1996; Wales 1996; Nayak et al 1997; Sun and Gong 1997), etc are aspects which require to be understood from a fundamental point of view. While free clusters of atoms have been identified experimentally, presence of such clusters in condensed state, with or without requiring a second medium to hold them together are also known. Of particular interest to this work are the clusters held together by a second medium, and are therefore subjected to the pressure exerted by the latter. This situation is often associated with the structure of glasses in which clusters generally possessing a high degree of order of the constituent atoms within themselves are surrounded by an amorphous tissue and are separated

\footnotetext{
*Author for correspondence

'Communication no. 1413 from Solid State and Structural Chemistry Unit.

${ }^{\ddagger}$ Refer to Z. Phys. D, Vol. 20 (1991) for a number of experimental studies.
}

from other similar clusters (Gaskell et al 1979; Bursill 1980). The nature of the pressure exerted by the surrounding tissue can cause both stabilization and deformation of these clusters. The formation of such clusters can be considered as resulting from frustrated growth of the nuclei formed during the nucleation step in supercooled melts. This is either due to enormously high viscosities of the medium or due to stabilization of the surfaces of the clusters due to thermodynamic reasons.

A few salient observations from the published studies on structure, dynamics and thermodynamics-especially the solid-liquid transition of free inert gas clusters are as follows (Etters and Kaelberer 1977; Jellinek et al 1986; Davis et al 1987; Labastie and Whetten 1990; Cheng et al 1992; Rose and Berry 1993): (i) The structures of small inert gas clusters are different from those of the bulk inert gas solids (face centred cubic structure is known to be the most stable in bulk) (Jellinek et al 1986; Davis et al 1987). (ii) The clusters are less stable and boil off at much lower temperatures (e.g. $\mathrm{Ar}_{13}$ cluster boils at around $36 \mathrm{~K}$ at zero pressure), losing their identity (Etters and Kaelberer 1977). (iii) They show a diffuse solid-liquid transition, which is not first order in the Ehrenfest sense (Etters and Kaelberer 1977; Jellinek et al 1986; Davis et al 1987; Labastie and Whetten 1990). (iv) They exhibit a solid-liquid co-existence (very different from that in the bulk), over a range of temperatures (for $\mathrm{Ar}_{13}$, the range is $29 \mathrm{~K}-36 \mathrm{~K}$ ). In this temperature range they keep flipping between solid and liquid phases, as the structural and energetic characterizations reveal (Labastie and Whetten 1990).

The interest in this work is to be able to do computer simulation studies on similar clusters and examine the effect of pressure experienced by the cluster due to the surrounding media on their structures and thermodynamics. Although realistic clusters may consist of 
hundreds of atoms, we examine here a 13-atom Ar cluster as the first step. We develop a method by which external pressure can be simulated through a plausible and heuristic pressure function. We have extended the method to examine the behaviour of next bigger cluster of $55 \mathrm{Ar}$ atoms. We have used both Monte Carlo (MC) (Allen and Tildesley 1987) and Nose's constant temperature molecular dynamics (NMD) (Nose 1984) methods in the present simulation. Important thermodynamic quantities have been calculated as a function of external pressure. The external pressure has been simulated by the use of a spherically symmetric potential described later. The simulation studies seem to provide some vital clues to the understanding of the phenomenon of glass transition.

\section{Simulation studies}

\subsection{Model potentials}

A Lennard-Jones interatomic potential has been used in this simulation and is given by,

$$
U^{\mathrm{int}}=\sum_{i<j=1}^{N} 4 \varepsilon\left(\left(\sigma / r_{i j}\right)^{12}-\left(\sigma / r_{i j}\right)^{6}\right)
$$

with $\varepsilon=120 k_{\mathrm{B}}$ and $\sigma=3.4 \AA$. The external potential is assumed to have the form,

$$
U^{\text {ext }}=\sum_{i=1}^{N} A \exp \left(B r_{i}^{2}\right)
$$

where $r_{i}$ is the distance of $i$ th atom from an initially assigned origin of coordinates, $B=0.001 / \sigma^{2}$ and $A$ the strength of the external potential, which is varied so as to produce the required external pressure on the system. The above form of external potential is dictated by the fact that the atoms on the surface experience a much higher force than those near the centre. The external and internal pressures, $P^{\text {ext }}$ and $P^{\text {int }}$ respectively, are defined as follows (Allen and Tildesley 1987),

$$
P^{\mathrm{ext}}=\sum_{i=1}^{N} \mathbf{r}_{i} \cdot \mathbf{F}_{i}^{\mathrm{ext}} / V
$$

where $\mathrm{F}_{i}^{\mathrm{ext}}$ is the external force on the $i$ th particle.

$$
P^{\text {int }}=W / V+(3 N-6) k_{\mathrm{B}} T / V,
$$

where, $W=\sum_{i<j=1}^{N} \mathbf{r}_{i j} \cdot \mathbf{F}_{i j} / 3$,

is the virial, $N$, the number of particles in the system, $k_{\mathrm{B}}$, the Boltzmann constant, $F_{i j}$, the force on $i$ th particle due to the $j$ th and $\mathbf{r}_{i j}=\mathbf{r}_{i}-\mathbf{r}_{j}$, where $\mathbf{r}_{i}$ and $\mathbf{r}_{j}$ are the position vectors to the $i$ th and $j$ th particles from the origin. $T$ is the temperature (instantaneous temperature in molecular dynamics (MD) and the temperature at which the sampling is done in the case of Monte Carlo (MC)). The time (or ensemble) averaged $\left\langle P^{\text {ext }}\right\rangle$ and $\left\langle P^{\text {int }}\right\rangle$ are expected to be equal at equilibrium.

Definition of cluster volume used in the expression for pressure is a major problem in these studies. This is due to the fact that the surfaces of small clusters are rather rough in comparison to their dimensions and use of continuum approximation to calculate their volumes is of doubtful validity. As a consequence the definition of pressures also becomes ambiguous. However, the instantaneous volume, $V$ of the cluster is defined following Cheng et al (1992) as

$$
V=\frac{4 \pi}{3}(d / 2)^{3},
$$

where $d$ is the largest value of the centre to centre separation of atoms in the cluster. We have tried to evaluate the appropriateness of this definition later, by comparing $\left\langle P^{\text {int }}\right\rangle$ and $\left\langle P^{\text {ext }}\right\rangle$ of the clusters, at various values of $\left\langle P^{\text {ext }}\right\rangle$ for different temperatures.

The total Hamiltonian of the system is therefore given as,

$$
H^{\mathrm{sys}}=\sum_{i=1}^{N} \frac{p_{i}^{2}}{2 m}+U^{\mathrm{int}}+U^{\mathrm{ext}}
$$

Extensive MC simulation (Allen and Tildesley 1987), has been performed over a wide range of temperatures $(5 \mathrm{~K}-95 \mathrm{~K})$ and pressures (20 atm.-1000 atm.). Nose's (1984) constant temperature molecular dynamics has also been performed over a smaller range of temperatures and pressures-mainly to check the correctness of results.

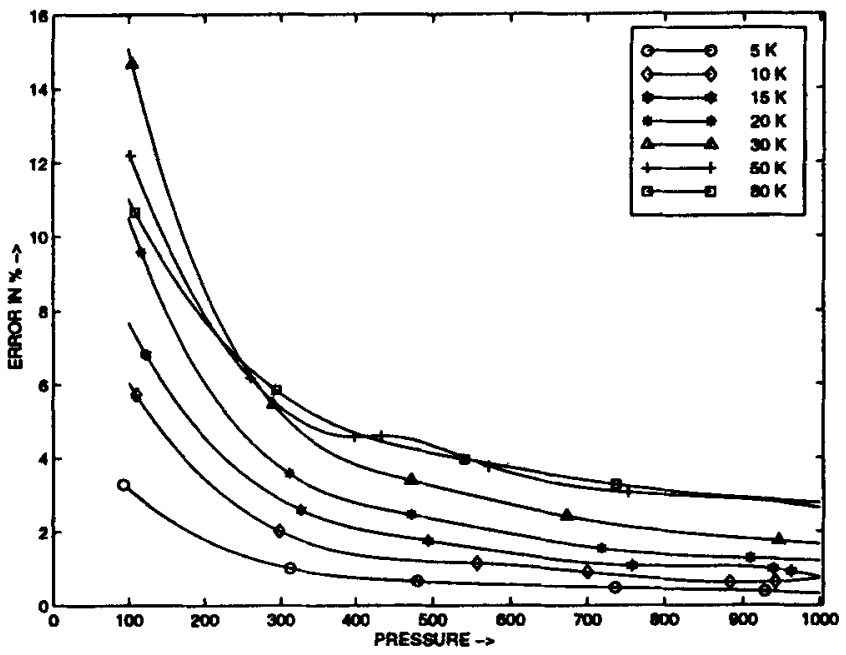

Figure 1. $\Delta P$ values as a function of $\left\langle P^{\text {ext }}\right\rangle$ (in atm.) for a few temperatures, for $\mathrm{Ar}_{13}$. Symbols represent actual data points and continuous lines are spline fits. 
Both the methods give canonical ensemble averages. The methods themselves are briefly described below.

(I) The MC method: The standard Metropolis method (Allen and Tildesley 1987) has been used with slight modifications in order to include the effect of the external potential on the system. The transition probability between states $n$ and $n+1$ in this case is given by,

$$
W_{n, n+1}=\exp \left[-\beta\left(U_{n+1}^{\text {tot }}-U_{n}^{\text {tot }}\right)\right]
$$

where, $U_{n}^{\text {tot }}=U_{n}^{\text {int }}+U_{n}^{\text {ext }}$ and $\beta=1 / k_{\mathrm{B}} T$.

The new state $n+1$ is accepted if, $W_{n, n+1} \geq \xi$ or rejected if, $W_{n, n+1}<\xi$, where $\xi$ is a random number between zero and one.

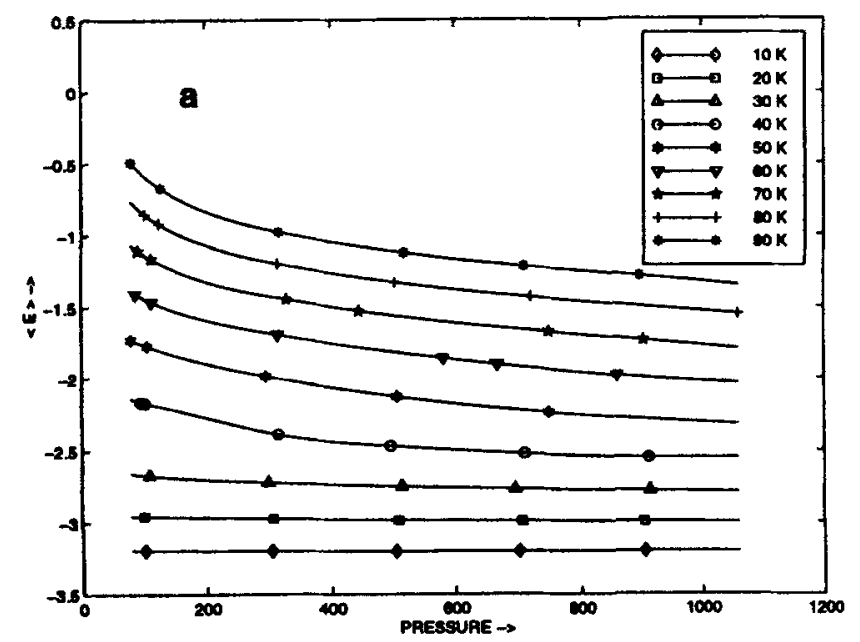

(II) The Nose's MD method: Nose's method makes use of an extended Hamiltonian given by,

$$
H^{\mathrm{extd}}=\sum_{i=1}^{N} \frac{p_{i}^{2}}{2 m s^{2}}+U^{\mathrm{tot}}+\frac{p_{s}^{2}}{2 Q}+(3 N-5) k_{\mathrm{B}} T \ln s,
$$

where $Q$ and $s$ may be interpreted as the mass and coordinate of the pseudo particle, which exchanges energy with the particles in the system. It has been shown that the MD (micro-canonical ensemble) average of any quantity given by the $H^{\text {extd }}$ is precisely the same as the canonical ensemble average given by $H^{\text {sys }}$ (Nose 1984). Also this extended Hamiltonian assures an average temperature $\langle T\rangle$ numerically equal to the required (input) temperature, $T$. In the present study, $Q$ was kept equal to $10 \sigma^{2}$ amu throughout.
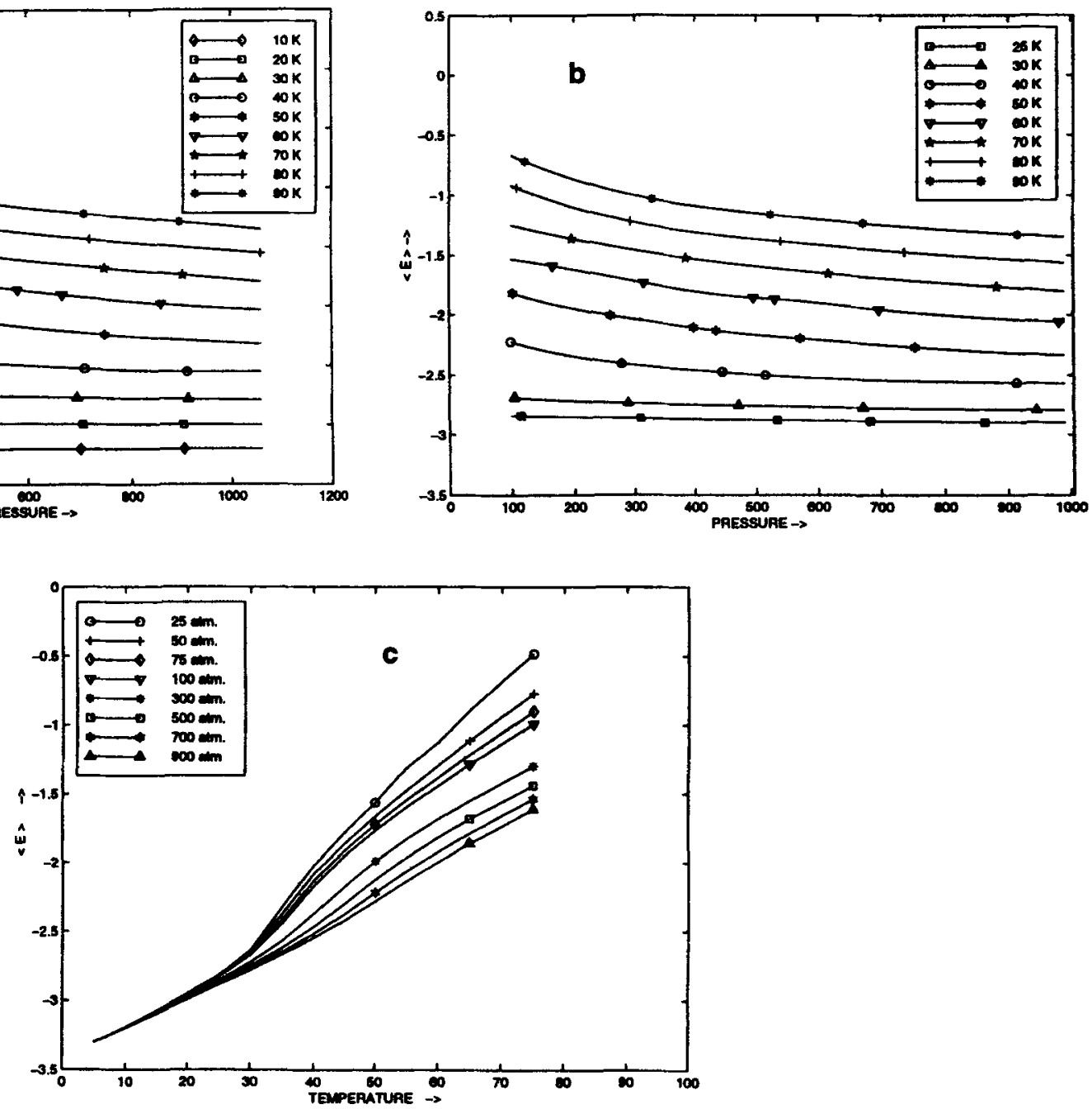

Figure 2. The average total internal energy (per atom, in units of $\varepsilon$ ) isotherms as a function of $\left\langle P^{\mathrm{ext}}\right\rangle$ (in atm.) (a) from $\mathrm{MC}$; (b) from NMD and (c) internal energy isobars generated from (a) as a function of temperature (in $\mathrm{K}$ ), for $\mathrm{Ar}_{13} . \mathrm{Symbols}$ in (a) and (b) represent simulation data. Symbols in (c) are only to identify the curves. 


\subsection{Details of simulation runs}

For the NMD studies we have used the algorithm suggested by Fox and Andersen (1984). We have chosen a time increment of $\sim 3 \mathrm{fs}$. Nearly $10^{6}$ steps were devoted for adjusting the parameter $A$, in the external pressure function in order to produce the required pressure on the system. The value of $A$ is held constant thereafter. The system is then equilibrated for further $2 \times 10^{6}$ steps, and averages are calculated for another $10^{7}$ steps. In the MC calculation also $10^{6}$ steps were devoted for pressure adjustment, $2 \times 10^{6}$ steps for equilibration and $5 \times 10^{6}$ steps for property averaging. Even then the present method does not offer good control of pressure on the system. Hence isotherms were generated (for average total internal energy, heat capacity and root mean square bond length fluctuation), at various temperatures. These isotherms were interpolated at required pressures, and isobars at various pressures were generated. Matlab (version 5.0.0.4064) cubic spline interpolation function was used for the above purpose. Simulation runs were carried out on IBM/580 work stations.

\section{Results and discussion}

The limitations arising from the definition of volume was first examined by calculating $\triangle P$, from NMD, which is defined as,

$$
\Delta P=\frac{\left\langle P^{\mathrm{int}}\right\rangle-\left\langle P^{\mathrm{ext}}\right\rangle}{\left\langle P^{\mathrm{int}}\right\rangle} \times 100 .
$$

In figure $1, \Delta P$ is plotted as a function of pressure at various temperatures. The observed discrepancy in the calculated $\left\langle P^{\text {int }}\right\rangle$ and $\left\langle P^{\text {ext }}\right\rangle$ can be attributed to the
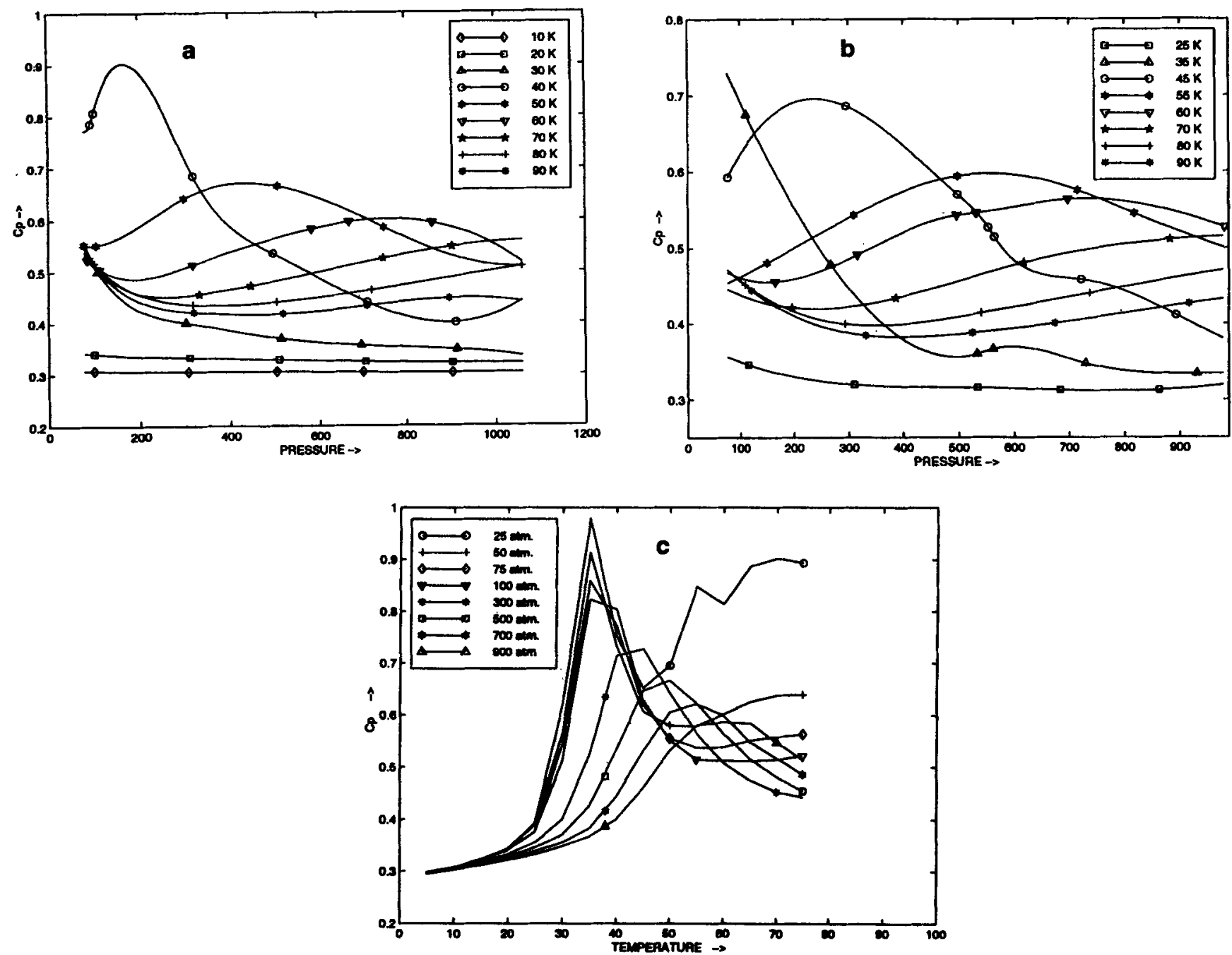

Figure 3. Heat capacity (in $\varepsilon \mathrm{K}^{-1}$ units) isotherms as a function of $\left\langle P^{\text {exi }}\right\rangle$ (in atm.) (a) from MC; (b) from NMD and (c) heat capacity isobars generated from (a) as a function of temperature (in $\mathrm{K}$ ), for $\mathrm{Ar}_{13}$. Symbols in (a) and (b) represent simulation data. Symbols in (c) are only to identify the curves. 
inadequate definition of volume. Though, $\Delta P$ keeps increasing with temperature, it decreases with increasing $\left\langle P^{\mathrm{ext}}\right\rangle$, which is encouraging. Since $P^{\mathrm{ext}}$ is not used directly in the subsequent calculations, we consider it as sufficiently accurate for examining the influence of external force on the cluster properties.

The total internal energy (the average value of $H^{\text {sys }}$ ) isotherms from MC and NMD are shown in figures $2 a$ and $2 b$. The isobars generated from figure $2 a$ are shown in figure $2 \mathrm{c}$. The heat capacities $\left(C_{\mathrm{P}}\right)$ were calculated from the total energy fluctuations as,

$$
C_{\mathrm{P}}=\frac{\left\langle U^{\mathrm{tot}^{2}}\right\rangle-\left\langle U^{\mathrm{tot}}\right\rangle^{2}}{k_{\mathrm{B}} T^{2}}
$$

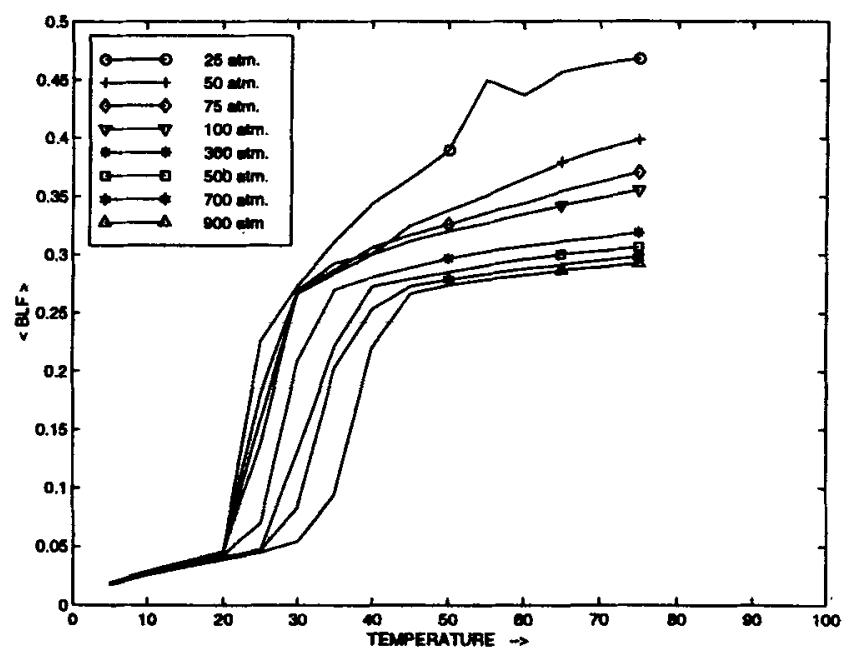

Figure 4. Average root mean square bond length fluctuation $(\langle B L F\rangle)$ as a function of temperature for various pressures (in atm.), for $\mathrm{Ar}_{13}$. Symbols are only to identify the curves.

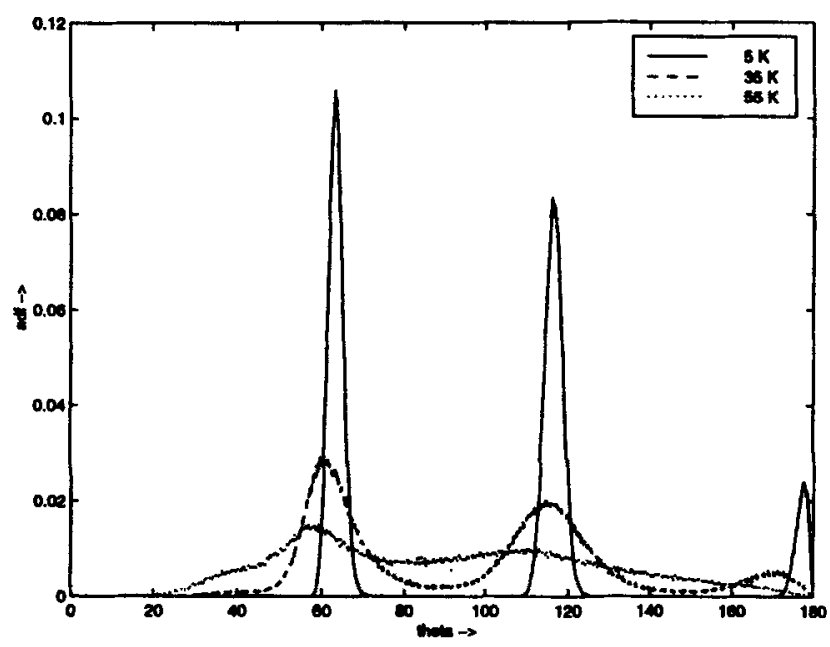

Figure 5. Angle distribution functions at $5 \mathrm{~K}, 35 \mathrm{~K}$ and $55 \mathrm{~K}$ at about $100 \mathrm{~atm}$.
The comparisons of heat capacity isotherms shown in figure 3a (from $M C$ ) and figure $3 b$ (from NMD) also reveal good agreement. As the pressure is increased the peak-heights lower and peak-widths broaden as seen in figure $3 \mathrm{c}$ (the $C_{\mathrm{P}}$ isobars are generated from figure 3a). This is a clear indication that the transition becomes more and more diffuse and smeared with increasing pressure. The shifts of the peaks also reveal that the solid-liquid (SL) transition temperature, $T_{t}$ increases with pressure.

The root mean square bond length fluctuation $\delta$, is a very useful quantity to locate solid-liquid transition temperature, $T_{\mathrm{t}}$ (Etters and Kaelberer 1977; Jellinek et al 1986; Davis et al 1987). $\delta$ is defined as,
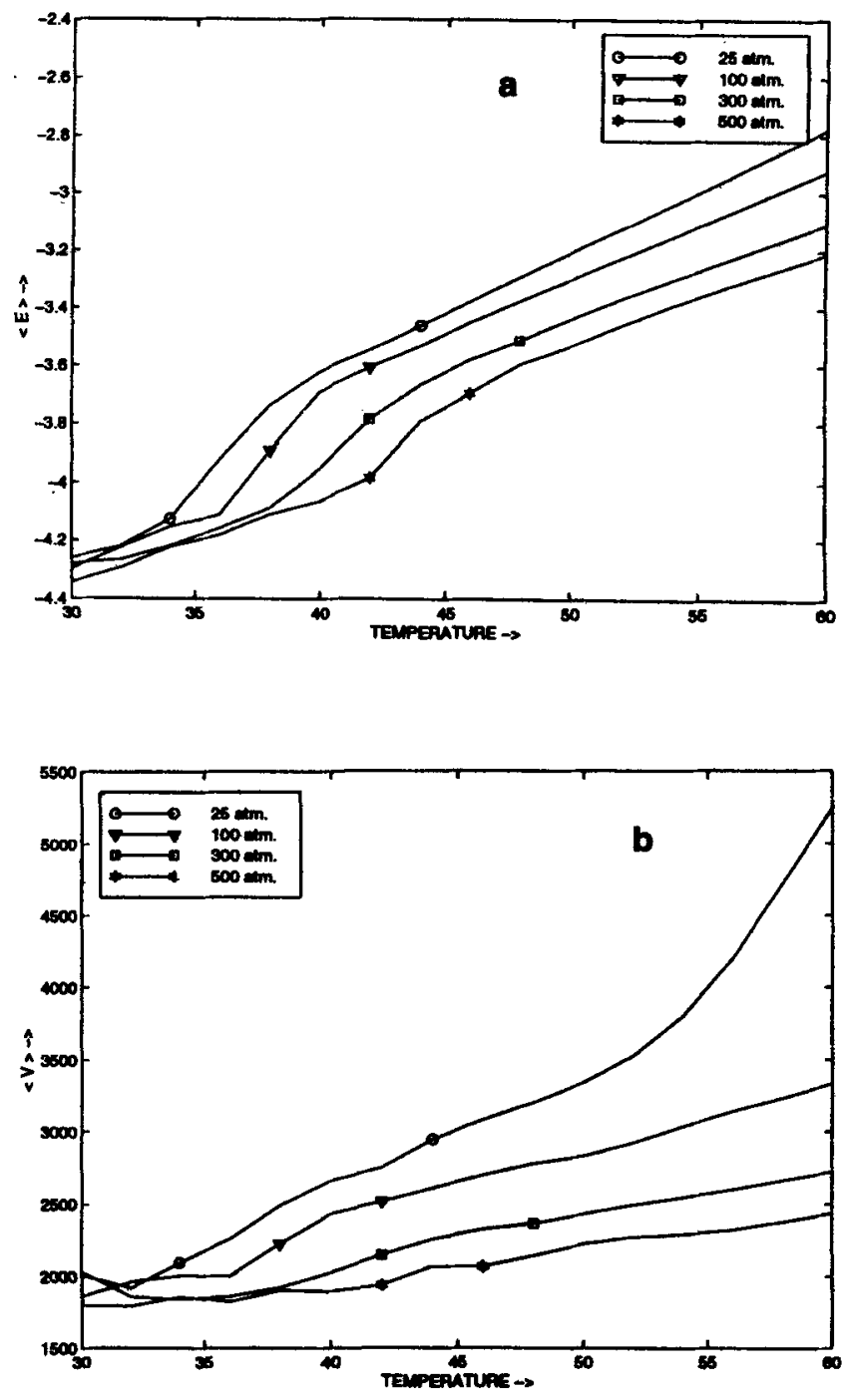

Figure 6. (a) The average total internal energy per atom (in units of $\varepsilon$ ) and (b) average volume (in $\AA^{3}$ ) as a function of temperature (in $K$ ) for various $\left\langle P^{\text {ext }}\right\rangle$ values for Arss. Symbols are only to identify the curves. 


$$
\delta=\frac{2}{N(N-1)} \sum_{i<j=1}^{N} \frac{\sqrt{\left\langle r_{i j}^{2}\right\rangle-\left\langle r_{i j}\right\rangle^{2}}}{\left\langle r_{i j}\right\rangle}
$$

$\delta$ exhibits a sharp change from 0.1 to 0.3 , in a narrow temperature range, around $T_{1}$ as shown in figure 4 . When $\delta \leq 0 \cdot 1$ it corresponds to a purely solid phase (so called Lindemann criterion) and when $\delta \geq 0.3$ it represents a purely liquid phase. The temperature range in which $\delta$ rises rapidly from 0.1 to 0.3 marks the co-existence of solid and liquid phases. It may be noted that $\delta$ is quite easy to calculate in simulation studies and its behaviour is somewhat insensitive to the details of the interaction potential. The $\delta$ calculated as a function of temperature at various pressures confirms that the peaks observed in $C_{\mathrm{P}}$ plots (figure 3c), indeed, correspond to the melting of the cluster and not to any solid-solid structural transition. It can be seen that $\delta$ tends to flatten out at values less than $0 \cdot 3$, at high pressures.

At low pressures (e.g. $25 \mathrm{~atm}$. and $50 \mathrm{~atm}$.) and at high temperatures (above $50 \mathrm{~K}$ ), yet another $C_{\mathrm{P}}$ peak (though very broad) emerges (figure $3 \mathrm{c}$ ). This is most likely due to the onset of liquid-gas transition of the cluster. Near liquid-gas transition a sharp change in $\delta$ has been reported in 2D systems by Rivera et al (1995). But we have not observed a sharp change of $\delta$ in this region.

Figure 5 shows the angle distribution function (ADF), calculated with respect to the central atom, using all triplets of atoms in the cluster for three different temperatures at about $100 \mathrm{~atm}$. pressure. There are peaks observed in ADF at $60^{\circ}, 120^{\circ}$ and $180^{\circ}$ and they correspond to the angles expected to be present in an icosahedral structure (Davis et al 1987). The application of isotropic pressure, therefore, appear to leave the icosa-

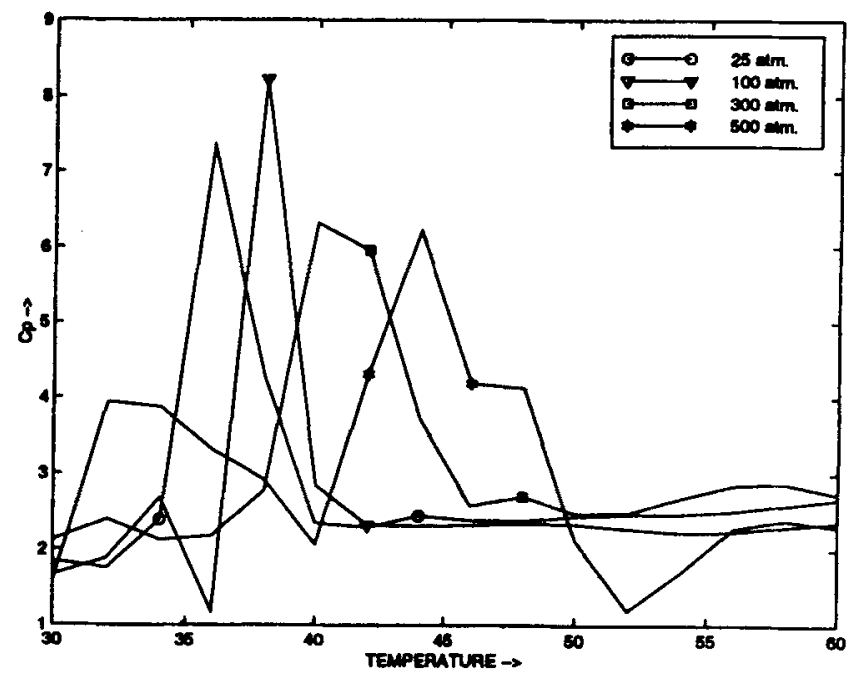

Figure 7. Heat capacity (in $\varepsilon \mathbf{K}^{-1}$ units) isobars as a function of temperature (in K) for $\mathrm{Ar}_{35}$. Symbols are only to identify the curves. hedral structure of the $\mathrm{Ar}_{13}$ cluster largely unaffected, till the melting temperature.

\subsection{The 55-atom Ar cluster}

Using the same interaction potential as employed for the 13-atom cluster, MC calculations were performed for a 55-atom argon cluster $\left(\mathrm{Ar}_{55}\right)$. The energies were computed for $15 \times 10^{6}$ steps in the transition region. The number of steps were reduced to $5 \times 10^{6}$ in the post transition region. Average energies, average volumes (figures $6 a$ and $6 b$, respectively) and heat capacities (figure 7) were evaluated at various pressures (from $25 \mathrm{~atm}$. to $500 \mathrm{~atm}$.) as a function of temperature. The heat capacity variations are shown only for a few pressures for the sake of clarity. It may be noted that variation of energy, volume and heat capacities is qualitatively similar to the variation of the same quantities for $\mathrm{Ar}_{13}$ cluster. The volume behaviour at $25 \mathrm{~atm}$. pressure is also suggestive of the liquid-vapour transition around $55 \mathrm{~K}$. The solid-liquid co-existence region and the bond length fluctuation behaviour are also similar for $\mathrm{Ar}_{55}$ cluster (figure 8).

Most important for the present discussion is the behaviour of heat capacities of the cluster for the various pressures. Although the peak heights seem to exhibit a slight increase at lower pressures, their heights decrease and the peaks broaden out at higher pressures. The heat capacity peaks shift to higher temperatures as the pressure is increased.

The transition temperature noted from the $C_{\mathrm{p}}$ peaks as a function of pressure, for $\mathrm{Ar}_{13}$ and $\mathrm{Ar}_{55}$ clusters, are shown in figure 9. At higher pressures the transition temperature of the $\mathrm{Ar}_{55}$ cluster is somewhat lower than that of $\mathrm{Ar}_{13}$ cluster. Also, the pressure coefficient of the transition temperature is slightly lower for the bigger cluster.

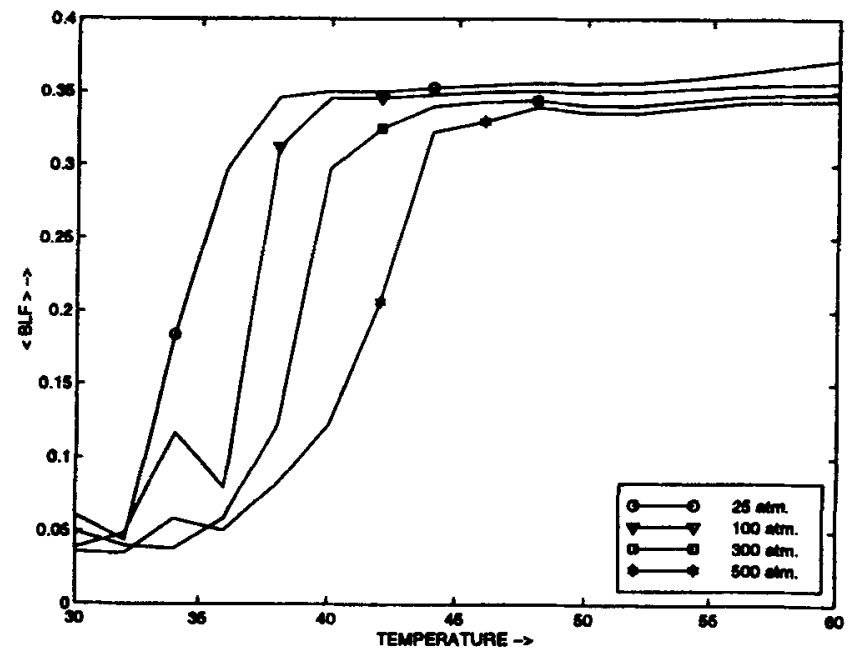

Figure 8. Average root mean square bond length fluctuation $(\langle B L F\rangle)$ as a function of temperature (in $\mathbf{K}$ ) for various $\left\langle P^{\text {ext }}\right\rangle$ values for Arss. Symbols are only to identify the curves. 
The compressibilities of the $\mathrm{Ar}_{13}$ and $\mathrm{Ar}_{55}$ clusters calculated from the volume fluctuations (Allen and Tildesley 1987) are $156 \times 10^{-11} \mathrm{~m}^{2} \mathrm{~N}^{-1}$ and $4942 \times 10^{-11}$ $\mathrm{m}^{2} \mathrm{~N}^{-1}$ (at $5 \mathrm{~K}$ and $25 \mathrm{~atm}$.) respectively. This may be compared to the experimental compressibility of bulk argon $98.8 \times 10^{-11} \mathrm{~m}^{2} \mathrm{~N}^{-1}$ (at $10 \mathrm{~K}$ and $0 \mathrm{~atm}$.) (Dobbs and Jones 1957). Although the compressibility of $\mathrm{Ar}_{55}$ cluster is significantly higher than that of $\mathrm{Ar}_{13}$, it is still surprising that the compressibilities of simulated clusters reflect reasonable magnitudes.

\subsection{Relevance to the glass transition phenomenon}

Cluster models of glass transition (Rao and Rao 1982; Parthasarathy et al 1983; Rao 1984) assume that glasses possess a cluster tissue texture, with clusters held in a matrix of the tissue. These clusters are generally assumed to be characterized by a greater topological order compared to that of the tissue, which is truly amorphous. The ordered arrangement of constituents in clusters has been evidenced in several lattice imaging studies (Gaskell et al 1979; Bursill et al 1980). These clusters generally are of the dimensions of several angstroms and therefore consist of several hundred atoms. It is possible in glasses where lattice fringes have not been observed in electron microscopic studies that the clusters are much smaller. The point of interest in this work is that in real glasses, clusters are subjected to the high pressures exerted by the surrounding amorphous matrix of the tissue.

The cluster model considers the glass transition as corresponding to the melting of the clusters, but they melt after the tissue itself melts. Since small clusters melt at characteristically lower temperatures, it would be more realistic to assume that clusters melt first and then the surrounding tissue dissolves into the molten clusters. In such a situation we should expect the variation of heat capacities of glasses to be dominated by the heat capacity variations of the clusters. Indeed the heat capacity behaviour during the melting of $\mathrm{Ar}_{13}$ cluster shown in figure $3 c$ is highly reminiscent of variations of heat capacity around $T_{\mathrm{g}}$ of most real glasses. We should therefore like to examine on a heuristic basis the implications of the pressure dependence of heat capacities of clusters to a possible origin of the glass transition in materials.

The $\mathrm{Ar}_{13}$ cluster has a volume of $506.9 \AA^{3}$ (at $5 \mathrm{~K}$ and $25 \mathrm{~atm}$.). In the bulk argon, volume occupied by 13 atoms of $A r$ is $486.59 \AA^{3}$ (at $0 \mathrm{~K}$ and $0 \mathrm{~atm}$.). In a hypothetical argon glass therefore a 13-atom cluster would have been subjected to a pressure by the matrix, whose magnitude is such that the cluster volume has decreased to its characteristic bulk volume. On the basis of the compressibility of $\mathrm{Ar}_{13}$ cluster, the pressure due to the matrix can be estimated as $267.2 \mathrm{~atm}$ (at $5 \mathrm{~K}$ ). But at such an external pressure (267.2 atm.), the $\mathrm{Ar}_{13}$ cluster is expected

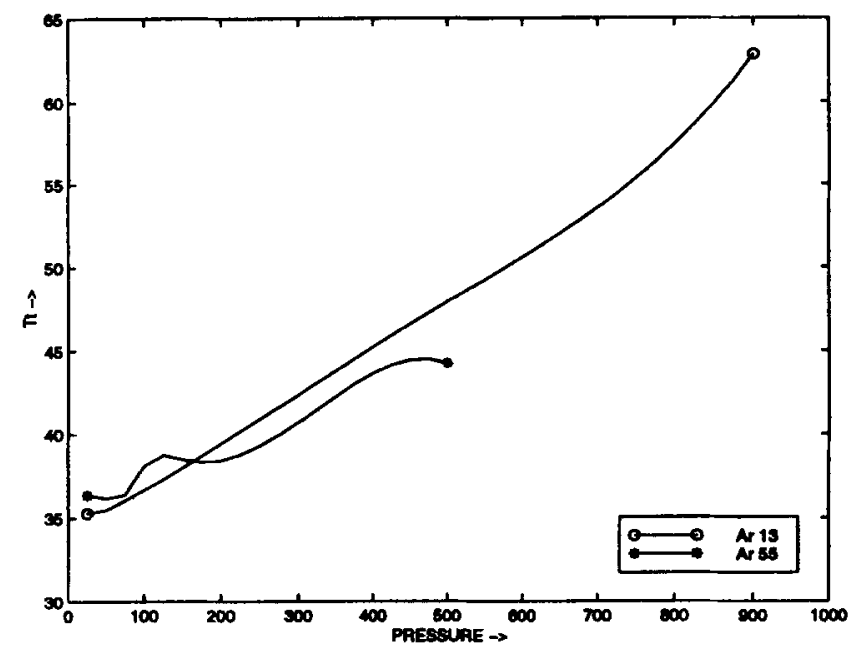

Figure 9. Melting points (in $\mathrm{K}$ ) of $\mathrm{Ar}_{13}$ and $\mathrm{Ar}_{55}$ as a function of $\left\langle P^{\text {ext }}\right\rangle$ (in atm.). Symbols are only to identify the curves.

to melt at an elevated temperatures of about $42 \mathrm{~K}$ (interpolated value from figure 9 ). The hypothetical argon glass should therefore exhibit a glass transition at $42 \mathrm{~K}$, and give rise to the typical $C_{\mathrm{P}}$ behaviour shown in figure 3c. Indeed $42 \mathrm{~K}$ is equal to $0.5 T_{\mathrm{m}}$, where $T_{\mathrm{m}}$ is the melting point $(84 \mathrm{~K})$ of solid argon. Thus $T_{\mathrm{g}} / T_{\mathrm{m}} \approx 0.5$, for the hypothetical glassy argon (Angell 1981). The value is suggestive of why argon does not form a glass easily. The compressibility of $\mathrm{Ar}_{55}$ cluster is somewhat anomalous and therefore a similar estimate of $T_{\mathrm{g}}$ was not attempted. This study therefore suggests that in all real glasses, the existence of a cluster-tissue texture with cluster volumes of the order of a few $\AA$ in radius are likely to be subjected to pressure in the range of a few 100 atmospheres, and their melting under pressure manifests as glass transition. This approach needs further examination by simulation studies on glass forming materials like $\mathrm{SiO}_{2}$.

\section{Acknowledgements}

One of the authors (PPK) is thankful to the Council of Scientific and Industrial Research (CSIR), New Delhi, India for fellowship. The authors also thank the Commission of the European Communities for financial support.

\section{References}

Allen M P and Tildesley D J 1987 Computer simulation of liquids (Oxford: Oxford University Press)

Angell C A 1981 in Annals of the New York Academy of Sciences (eds) J M O'Reilly and M Goldstein 371 p. 136

Bursill L, Thomas J M and Rao K J 1980 Nature 289157

Cheng Hai-Ping, Li Xiuling, Whetten R L and Berry R S 1992

Phys. Rev. A46 791

Chitra R and Yashonath S 1996 Chem. Phys. Letts. 252384 
Davis H L, Jellinek J and Berry R S 1987 J. Chem. Phys. 86 6456

Dobbs E R and Jones G O 1957 Rep. Prog. Phys. 20516

Etters R D and Kaelberer J 1977 J. Chem. Phys. 665112

Frantz D D 1995 J. Chem. Phys. 1023747

Fox J R and Andersen H C 1984 J. Phys. Chem. 884019

Gaskell P H, Smith D J, Catto C J D and Cleaver J R A 1979 Nature 281465

Häkkinen H and Manninen M 1996 Phys. Rev. Letts 761599

Ikesyoji Tamio, Hafskjold Bjørn, Hashi Yuichi and Kawazoe Yoshiyuki 1996 J. Chem. Phys. 225126

Jellinek J, Beck T L and Berry R S 1986 J. Chem. Phys. 84 2783
Labastie P and Whetten R L 1990 Phys. Rev. Letts 651567

Nayak S K, Jena P, Stepanyuk V S, Hergert W and Wildberger K 1997 Phys. Rev. B56 6952

Nose S 1984 Mol. Phys. 52255

Parthasarathy R, Rao K J and Rao C N R 1983 Chem. Soc. Rev. 12361

Rao K J 1984 Proc. Indian Acad. Sci. (Chem. Sci.) 93389

Rao K J and Rao C N R 1982 Mater. Res. Bull. 171337

Rivera Y, Weber D C and Lopez G E 1995 J. Chem. Phys. 103 10627

Rose J P and Berry R S 1993 J. Chem. Phys. 983246

Sun D Y and Gong X G 1997 J. Phys.: Cond. Matter 910555

Wales D J 1996 Science 271925 